\title{
Estados claves en el comercio intracomunitario del tomate en la Unión Europea
}

\author{
J. de Pablo ${ }^{1, *}$, M.A. Giacinti² y T. García ${ }^{3}$
}

1 Departamento de Economía y Empresa. Universidad de Almería. Carretera de Sacramento s/n, 04120 Almería, España

2 Centro de Información Frutihortícola (www.cif-businessintelligence.com) y Gabinete MAG. Centenario s/n, Q8309 Neuquén, Argentina

3 Instituto de Economía, Geografía y Demografía del Consejo Superior de Investigaciones Científicas. Albasanz, 26-28, 28037 Madrid, España. Solvay Brussels School of Economics and Management. Avenue Franklin Roosevelt 42, 1050 Brussel, Bélgica

\section{Resumen}

El presente artículo tiene como objetivo identificar que Estados miembros resultan claves para el comercio intracomunitario del tomate en la Unión Europea para los años 2007-2013. Se va a utilizar la metodología del análisis input-output y para ello se van a elaborar dos tablas de transacciones comerciales intersectoriales, una para 2007 y otra para 2013. A partir de las mismas se realizaran las tablas de coeficientes técnicos e inversas. Para estudiar los efectos directos de los encadenamientos se obtendrán los valores de los coeficientes de Streit y de Chenery-Watanabe. Para finalizar se incorporaran los efectos indirectos por medio del coeficiente de Rasmussen. Los resultados señalan como "claves" a los Países Bajos y Bélgica, que precisamente basan su estrategia en la reexportación. España, a pesar de ser el segundo exportador de tomate de la Unión Europea habría dejado de tener la consideración de "Estado clave" en 2013.

Palabras clave: Análisis input-output, interdependencia, encadenamientos.

\section{Abstract \\ Key States in intra-EU tomato trade}

This paper identifies which are the key Member States for the intra-Community trade of tomatoes in the European Union in the period 2007-2013. We apply the input-output analysis methodology using two tables of commercial transactions across sectors. We calculate/compute technical coefficients, the inverse matrix and, in order to study the productive chain, we obtain the Streit and Chenery Watanabe's coefficients. Finally indirect effects are also incorporated through the Rasmussen coefficients. The results show that the Netherlands and Belgium are the 'key countries' in the tomato market, countries that have a specific strategy based on re-export. Besides this, Spain, the second tomato exporter of the European Union, is no longer considered a "key country" in 2013.

Key words: Input-output analysis, interdependence, linkages.

* Autor para correspondencia: jdepablo@ual.es https://doi.org/10.12706/itea.2016.028 


\section{Introducción}

Las tablas input-output son un instrumento habitual utilizado por las autoridades económicas para analizar el funcionamiento de sus sectores productivos tanto en su vertiente estructural como coyuntural. La aplicación de estas tablas es muy diversa. En su origen, el Tableau Économique de Quesnay (Miller y Blair, 1985) demostraba que la vida económica funcionaba como una máquina, como un organismo vivo. Posteriormente, Leontief (1941) utilizó la tabla input-output para analizar la estructura productiva de los Estados Unidos. Temas más recientes son los relacionados con el consumo energético (Bojacá et al., 2012), el medioambiente (Hoekstra et al., 2014) o la contabilidad social (Keuning y Ruijter, 1988), entre otros.

La medición del estudio de las relaciones de dependencia entre los sectores se inicia con las denominadas ligazones o eslabonamientos. Estos conceptos fueron introducidos por Hirschman (1958) en sus estudios sobre la economía del desarrollo. La dirección y el peso de las ligazones sectoriales indican la capacidad potencial de un sector para estimular a otros, por lo que la concentración de recursos en aquellas actividades con mayores ligazones impulsaría más rápidamente el crecimiento que la dedicación de dichos recursos a otras actividades.

Sobre países claves podemos destacar los artículos de Andreososso y Yue (2004) para China; Gülsün y Keçeli (2009) para Turquía; Olczyk (2011) para Polonia y Jahangard y Keshtvarz (2012) para Irán, Corea del Sur y Turquía.

El presente trabajo se centra en el comercio exterior, y en concreto en las relaciones de interdependencia (exportaciones/importaciones) que tienen los distintos Estados miembros de la Unión Europea (U.E.) en un producto específico como es el tomate. Se pretende conocer si alguno de los Estados puede ser considerado "clave" en cuanto a sus ligazones y si entre los años 2007-2013 ha supuesto cam- bios importantes en su jerarquización. Se ha escogido como año de partida para nuestro análisis el 2007 que corresponde con el inicio del cambio de ciclo económico en Europa.

El tomate es la primera hortaliza comercializada en Europa y tiene una importancia significativa en varios Estados miembros, tanto en su aspecto de producción como de comercialización y consumo. Está siendo, además, objeto de una considerable competencia por parte de países terceros, sobre todo en los meses de invierno.

\section{Comercio intra/extra comunitario del tomate en la U.E.}

De acuerdo al cuadro 1, los ratios entre el comercio intracomunitario y el extracomunitario han seguido pautas divergentes. Mientras que la relación entre las importaciones extracomunitarias respecto a las intracomunitarias ha descendido, las exportaciones extracomunitarias respecto a las intracomunitarias han aumentado. Francia presenta un valor mayor en cuanto a la relación de sus importaciones extracomunitarias/ intracomunitarias y en general se aprecia que los principales exportadores (Países Bajos, España y Francia) han incrementado ese ratio.

Polonia destaca en la relación entre las exportaciones extracomunitarias/ intracomunitarias con el mayor valor. Como se verá más adelante, se debe tanto a sus ventas al mercado ruso como a su papel como reexportador a otros países.

\section{Importaciones comunitarias}

El principal país tercero exportador a la Unión es Marruecos, seguido de lejos por Turquía. Como se aprecia en el cuadro 2, entre los dos, suponen aproximadamente el $83 \%$ de las importaciones en el año 2013. 
Cuadro 1. Relación entre las importaciones/exportaciones extracomunitarias respecto a las importaciones/exportaciones intracomunitarias (\%)

Table 1. Relationship between imports / exports extra EU and imports / exports intra EU (\%)

\begin{tabular}{lrrrr}
\hline & Importaciones extra/ & intracomunitarias & Exportaciones extra/intracomunitaria \\
\hline Países Bajos & 2007 & 2013 & 2007 & 2013 \\
España & 8,21 & 13,13 & 6,87 & 6,86 \\
Francia & 11,70 & 25,22 & 2,75 & 5,95 \\
Bélgica & 125,02 & 117,57 & 7,44 & 5,49 \\
Italia & 5,47 & 2,13 & 14,98 & 11,36 \\
Alemania & 3,63 & 1,42 & 6,12 & 8,96 \\
Portugal & 0,35 & 0,24 & 4,19 & 5,08 \\
Polonia & 0,00 & 1,72 & 0,04 & 0,07 \\
Grecia & 4,69 & 1,94 & 21,33 & 308,84 \\
Reino Unido & 53,68 & 82,82 & 125,08 & 10,74 \\
Otros & 2,69 & 11,50 & 7,36 & 60,88 \\
Total & 31,18 & 6,84 & 57,97 & 263,03 \\
\hline
\end{tabular}

Fuente: Elaboración propia a partir de Eurostat.

Cuadro 2. Importaciones de países terceros a la Unión Europa (miles de Tm) Table 2. Imports from third countries to the European Union (thousands of tons)

\begin{tabular}{lrrlrr}
\hline País & 2007 & $\%$ & País & 2013 & $\%$ \\
\hline Marruecos & 302391 & 63,41 & Marruecos & 365694 & 82,89 \\
Turquía & 94224 & 19,76 & Turquía & 29310 & 6,64 \\
Israel & 26762 & 5,61 & Senegal & 10379 & 2,35 \\
Macedonia & 21346 & 4,48 & Túnez & 10217 & 2,32 \\
Siria & 10956 & 2,30 & Macedonia & 9299 & 2,11 \\
Senegal & 7299 & 1,53 & Israel & 6440 & 1,46 \\
Jordania & 3230 & 0,68 & Albania & 3983 & 0,90 \\
Túnez & 3160 & 0,66 & República Dominicana & 1801 & 0,41 \\
Serbia & 2877 & 0,60 & Egipto & 1601 & 0,36 \\
Otros & 4617 & 0,97 & Otros & 2467 & 0,56 \\
Total & 476862 & 100 & Total & 441191 & 100 \\
\hline
\end{tabular}

Fuente: Elaboración propia a partir Eurostat. 
De la información suministrada por el cuadro anterior, se aprecia:

- La puerta de entrada del tomate alauita es Francia (concretamente el mercado de Saint-Charles en Perpiñán) aunque luego hay reexportaciones a otros Estados miembros. Supone aproximadamente el $80 \%$ de las exportaciones del Reino Alauita. El resto se envía al Reino Unido, España y los Países Bajos.

- Turquía centra sus exportaciones en Rumanía y Bulgaria con un $67 \%$ de sus exportaciones. Otros mercados significativos son Polonia, Croacia y Países Bajos.

- A otro nivel inferior están Senegal y Túnez, pero se aprecia un aumento en su participación.

- La República de Macedonia e Israel son exportadores tradicionales a la U.E.
- Analizando su evolución se aprecia como Marruecos es el líder incuestionable y Turquía va perdiendo importancia. El precio de entrada preferencial del que disfruta Marruecos con su acuerdo con la Unión Europea y la reducción significativa de los derechos de aduana para el tomate fuera de cuota, representan dos ventajas comparativas importantes para el reino alauita que este ha sabido movilizar. La existencia de relaciones comerciales tradicionales con operadores franceses (y españoles más recientemente) es otro factor que explica esta divergencia de comportamiento entre ambos suministradores.

\section{Exportaciones comunitarias}

El cuadro 3 recoge los principales destinos de las exportaciones comunitarias de tomate.

Cuadro 3. Exportaciones de la Unión Europea a terceros países. Miles de Tm (2007-2013)

Table 3. Export from third countries to the European Union. Thousands of tons (2007-2013)

\begin{tabular}{lrrlrr}
\hline Países & 2007 & $\%$ & Países & 2013 & $\%$ \\
\hline Rusia & 96385 & 56,60 & Rusia & 229584 & 63,10 \\
Suiza & 19178 & 11,26 & Bielorrusia & 52884 & 14,53 \\
Noruega & 18887 & 11,09 & Suiza & 28238 & 7,76 \\
Ucrania & 9164 & 5,38 & Noruega & 23748 & 6,53 \\
Bielorrusia & 8228 & 4,83 & Ucrania & 12349 & 3,39 \\
Estados Unidos & 6507 & 3,82 & Emiratos Árabes & 3464 & 0,95 \\
Albania & 3010 & 1,77 & Serbia & 2597 & 0,71 \\
Otros & 9930 & 5,83 & Otros & 10995 & 3,02 \\
Total & 170286 & 100 & Total & 363859 & 100 \\
\hline
\end{tabular}

Fuente: Elaboración propia a partir Eurostat.

Entre otras conclusiones cabría destacar las siguientes:

- Rusia era en el 2013 el principal mercado a la exportación, seguido de Bielorrusia.
Estos dos países han incrementado notablemente sus compras en los años de estudio. Sus principales proveedores directos son Lituania y Polonia. La diferencia entre 
ellos es que el primero no es productor y el segundo si lo es: exporta en verano y reexporta en invierno. Se comprende porque el embargo decretado por la Federación Rusa en relación a las frutas y hortalizas haya tenido un impacto directo tan importante en estos dos Estados. España y los Países Bajos también tienen significación como proveedores tanto para Rusia como Bielorrusia.

- Suiza y Noruega también son importadores tradicionales de tomate europeo. Sus proveedores son, en el caso de Suiza, Francia, España, Italia y los Países Bajos. Este último domina el mercado noruego.

\section{Material y métodos}

A partir de la realización de una tabla de transacciones comerciales intersectoriales entre los distintos Estados miembros en los años 2007 y 2013, se va a efectuar la explotación de la misma utilizando el análisis input-output.

Se parte de la tabla de transacciones comerciales que resume las relaciones entre los distintos países $\left(x_{i j}\right)$. Las columnas presentan las importaciones (toneladas) y las filas las exportaciones (toneladas). La suma total de las importaciones intracomunitarias coincide con el de las exportaciones intracomunitarias como se puede apreciar en los cuadros cuadro 4 y 5 . Los datos han sido obtenidos de Eurostat (Comisión Europea, 2014a) y de los informes del grupo europeo del tomate (Comisión Europea 2014b).

Una vez obtenida la tabla de transacciones comerciales se va a proceder a la obtención de la tabla de coeficientes técnicos. Los componentes de esta tabla representan el esfuerzo comercial directo que lleva a cabo el país i por unidad de consumo del país j. El valor de cada uno de los coeficientes técnicos sería:

$$
a_{i j}=\frac{x_{i j}}{c_{i j}}
$$

Para la obtención de la matriz de coeficientes técnicos se necesita el consumo aparente (CA) de los distintos países que es igual a la producción $(\mathrm{P})$ más las importaciones intracomunitarias (II) menos las exportaciones intracomunitarias (EI) más las importaciones extracomunitarias (IE) menos las exportaciones extracomunitarias (EE) tal y como se puede observar en el cuadro 6.

Las tablas de los coeficientes técnicos se reflejan en los cuadros 7 y 8.

A partir de la matriz de coeficientes técnicos se va a proceder a obtener la matriz tecnológica que es la diferencia entre la matriz identidad y la de coeficientes técnicos.

Con el resultado de la matriz se obtendrá la inversa de la misma. Esta matriz representa las relaciones directas e indirectas entre consumo y flujos comerciales de los países i y j. En este caso sus valores se observan en los cuadros 9 y 10.

Las distintas matrices logradas nos van a dar información sobre las relaciones directas e indirectas entre los Estados miembros.

A través de la matriz de transacciones se van a obtener las relaciones directas, y se van a utilizar dos aplicaciones clásicas del análisis input-output, los coeficientes de Streit (1969) y de Chenery-Watanabe (1958).

El coeficiente de Streit mide las relaciones comerciales entre dos países. Y se consigue al ponderar las ligazones directas de un país hacia atrás (importaciones) y las ligazones directas hacia adelante (exportaciones) de los países i y j.

Las ligazones hacia atrás de un Estado j con respecto a otro i nos viene dada por la razón entre las importaciones que $\mathrm{j}$ hace de $\mathrm{i}$ y el volumen de importaciones totales $\mathrm{j}$.

$$
p_{i j}=\frac{X_{i j}}{\sum_{i=1}^{n} X_{i j}}
$$




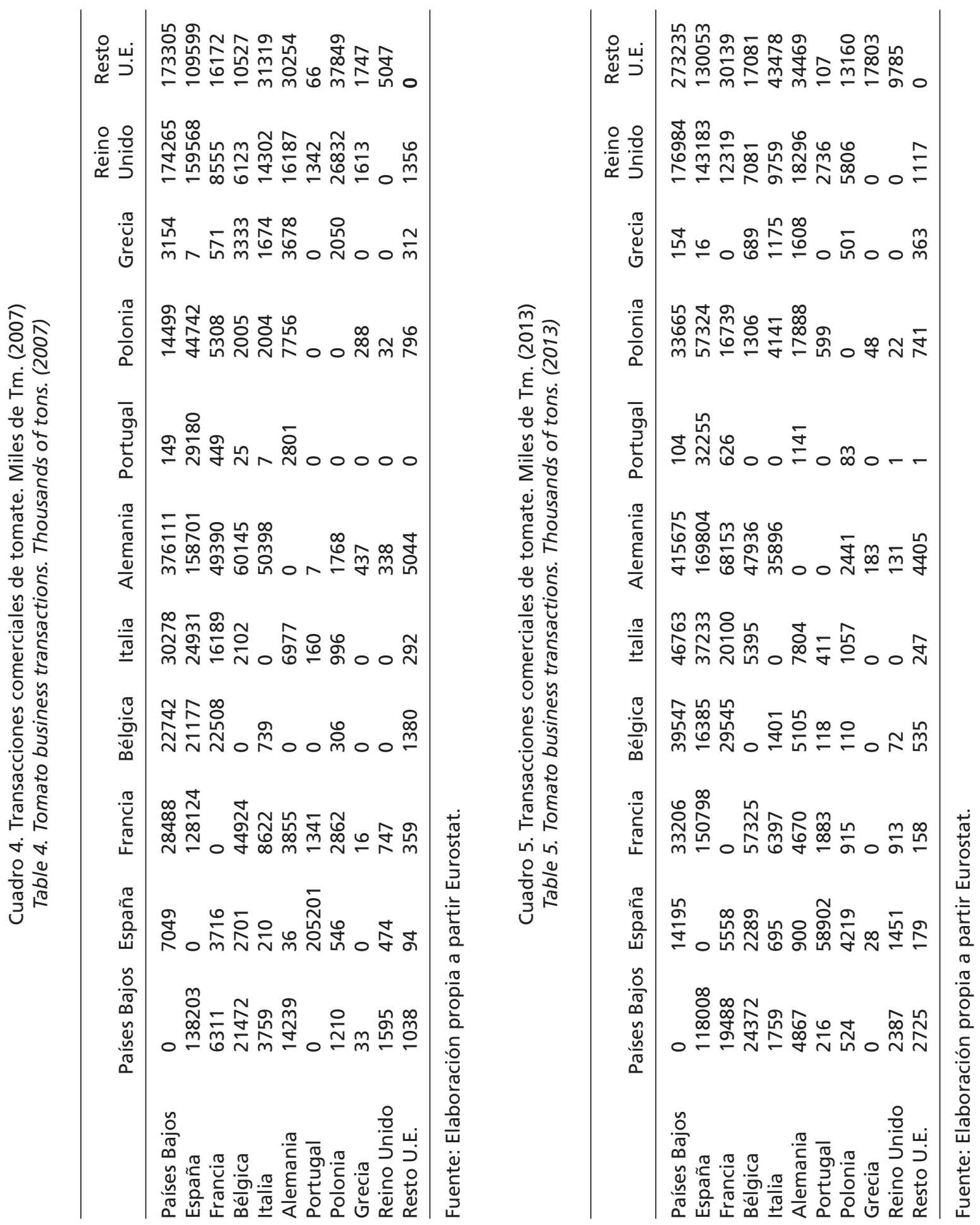




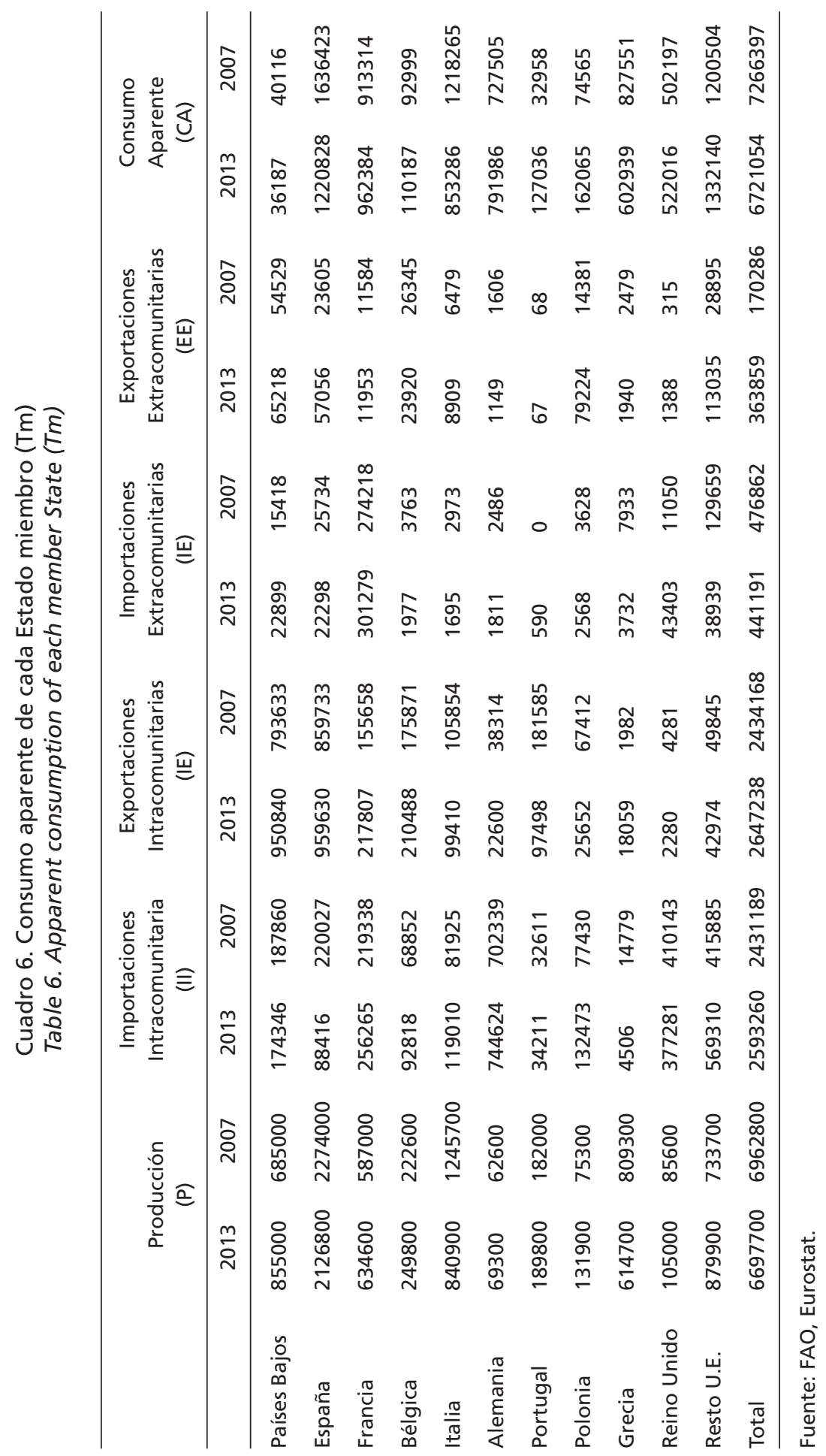




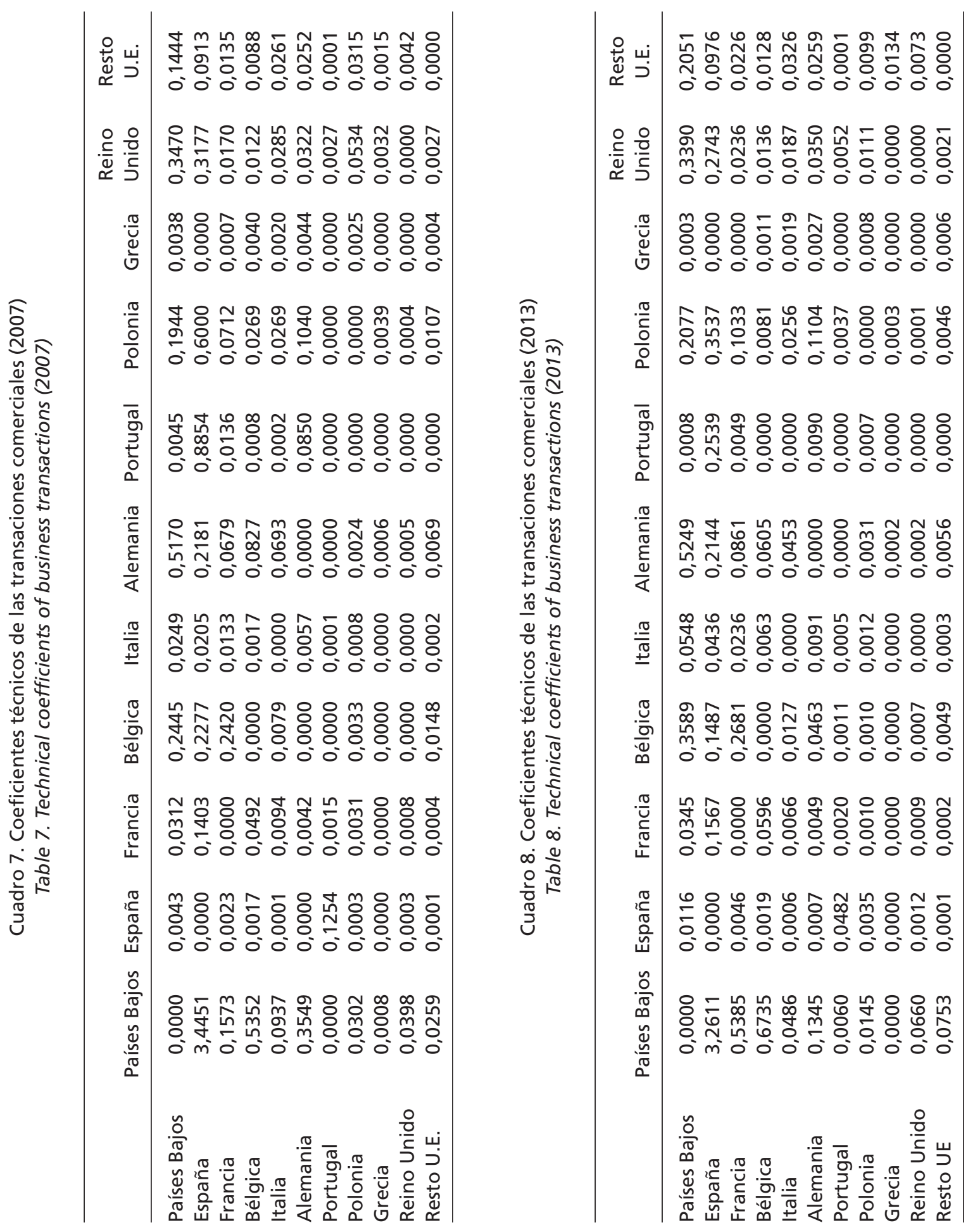



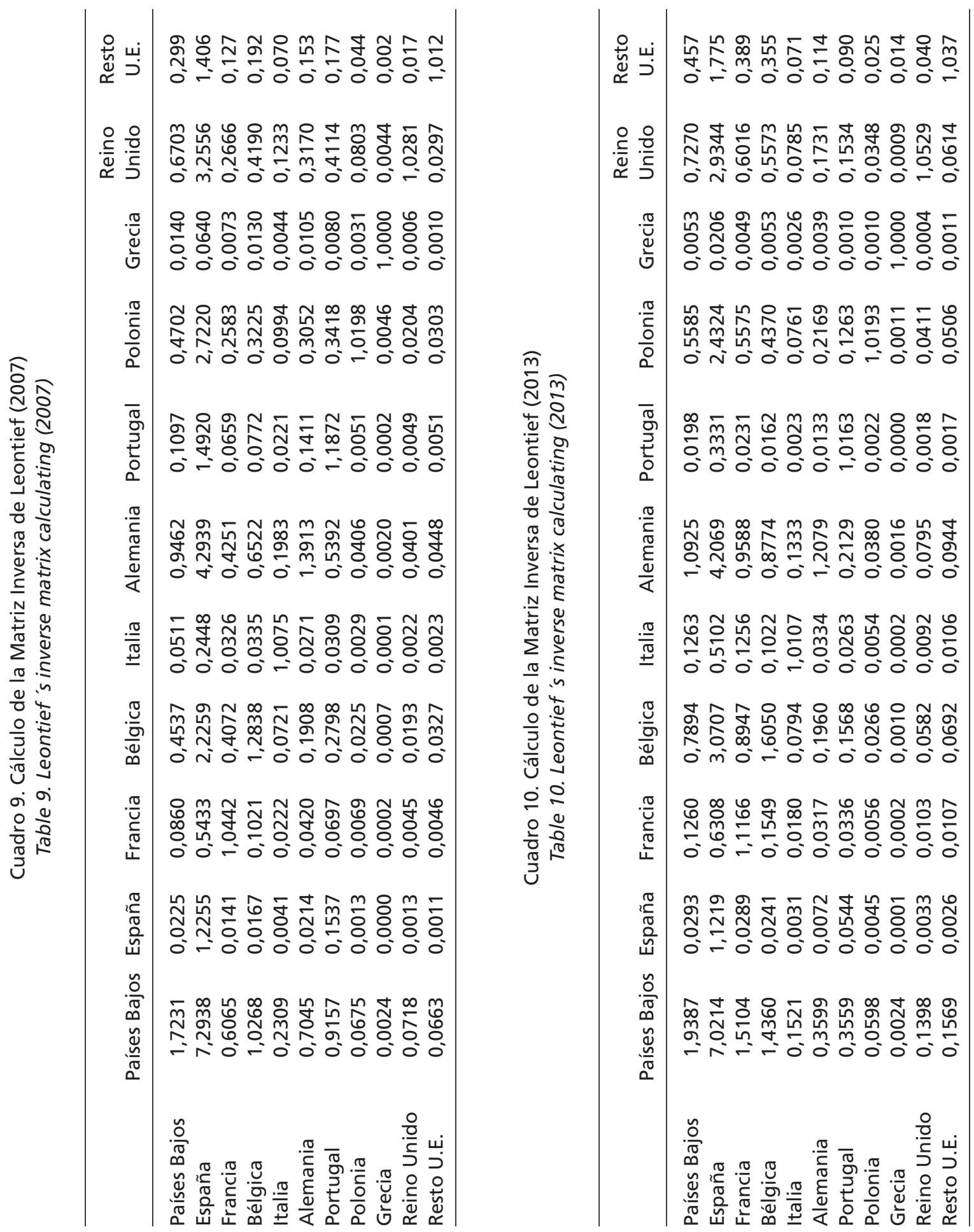
Las ligazones hacia delante de i con $\mathrm{j}$ nos vendrán dados igualmente por el volumen de exportaciones de i a j y las exportaciones totales de i.

$$
q_{i j}=\frac{X_{i j}}{\sum_{j=1}^{n} X_{i j}}
$$

A partir de estos valores se consigue el coeficiente de Streit, que es igual a la media ponderada de los cuatro coeficientes anteriores.

$S T_{i j}=S T_{j i}=\frac{1}{4}\left(\frac{x_{i j}}{\sum_{j=1}^{n} X_{i j}}+\frac{x_{i j}}{\sum_{i=1}^{n} X_{i j}}+\frac{X_{j i}}{\sum_{j=1}^{n} X_{i j}}+\frac{X_{j i}}{\sum_{i=1}^{n} X_{i j}}\right)$

El resultado debe de interpretarse como un valor promedio que nos indica el peso que tienen las transacciones directas entre dos Estados cualesquiera con respecto a las importaciones y exportaciones intracomunitarias del sistema. Una vez obtenidos los valores se podrá llevar a cabo una jerarquización de las relaciones entre los países estudiados.

Para elaborar una jerarquización de la capacidad de arrastre por países individualizado se van a usar los coeficientes de Chenery-Watanabe, que se definen de la siguiente manera:

- Eslabonamientos anteriores o ligazones de las importaciones. Miden la capacidad de un país de arrastrar directamente a otros ligados a él, por su importación de tomates $y$, estimulando, a su vez, la actividad de tales Estados:

$$
P_{j}=\frac{\sum_{i=1}^{n} x_{i j}}{x_{j}}(j=1,2, \ldots, n)
$$

- Eslabonamientos posteriores o ligazones de las exportaciones. Miden la capacidad de un Estado de estimular el consumo a otros, en virtud de su capacidad de exportar:

$$
Q_{i}=\frac{\sum_{j=1}^{n} x_{i j}}{x_{i}}(i=1,2, \ldots, n)
$$

Los coeficientes de Chenery-Watanabe presentan unas limitaciones importantes (Jahangard y Keshtvarz, 2012): a) Utilizan los coeficientes directos de la matriz de transacciones sin tener en cuenta los efectos indirectos.

b) Los coeficientes obtenidos representan medidas sin consideración alguna de sus desviaciones.

c) Estos índices no son ponderados.

La matriz inversa nos da información sobre el grado de interdependencia de los componentes del sistema y calcula los efectos totales (directos e indirectos) de un Estado con respecto a los otros. A partir de esta matriz se van a obtener los siguientes coeficientes: interno, de absorción, de difusión y Rasmussem. De esta manera se analiza si hay algún Estado clave tomando en consideración los efectos totales.

Los coeficientes internos corresponden a los valores de la diagonal principal de la matriz inversa y representan el esfuerzo total que debe de realizar un país ante el incremento en una unidad de su demanda final.

Los coeficientes de efectos difusión y absorción se pueden definir como:

El primero señala el efecto final sobre todos los países de un incremento de una unidad en la demanda final del país $j$.

$$
E D_{j}=\sum_{i=1}^{n} A_{i j}
$$

El segundo define el efecto final sobre un país $i$ cuando se produce un incremento de una unidad en la demanda final de todos los países.

$$
E A_{i}=\sum_{j=1}^{n} A_{i j}
$$

Reformulando los efectos difusión y absorción, se obtienen los coeficientes de Rasmussen denominados de poder y sensibilidad de dispersión. Estos coeficientes se utilizan para diferenciar las características de las ramas en función de sus efectos de arrastres. Y mejoran a los de Chenery Watanabe en los tres aspectos que eran limitantes para estos. 
El coeficiente de poder de dispersión $\left(u_{j}\right)$ describe la extensión relativa sobre la que un aumento de la demanda final de tomate del país $j$ se dispersa a través del sistema de países de la U.E. En otras palabras, es la extensión o alcance que una expansión de un país j provoca sobre el sistema. Su expresión matemática es la siguiente:

$$
u_{j}=\frac{\sum_{i=1}^{n} \frac{A_{i j}}{n}}{\sum_{i=1}^{n} \sum_{j=1}^{n} \frac{A_{i j}}{n^{2}}}
$$

Donde el numerador es la proporción del multiplicador de la producción por país (no ponderado), y el denominador, la media global (promedio de las medias de países) que sirve para normalizar los resultados y facilitar las comparaciones entre los países.

Si $u_{j}>1$, significa que los requisitos de importaciones intermedias generados por un aumento unitario de la demanda final del país $j$ ésimo son mayores para este país que para la media de la economía y, por lo tanto, se trata de un país con un fuerte poder relativo de arrastre hacia atrás sobre el sistema productivo.

El coeficiente de sensibilidad de dispersión $\left(u_{i}\right)$ expresa la extensión o medida en que el sistema de países pesa sobre el país i. En otros términos, es la medida en que el país $i$ es afectado por una expansión en el sistema.

$$
u_{i}=\frac{\sum_{j=1}^{n} \frac{A_{i j}}{n}}{\sum_{i=1}^{n} \sum_{j=1}^{n} \frac{A_{i j}}{n^{2}}}
$$

Donde el numerador es la proporción del multiplicador de una expansión uniforme de la demanda por el país (no ponderado), y el denominador, la media global.

$\mathrm{u}_{\mathrm{i}}>1$ indica que el país $i$-ésimo expande sus exportaciones intermedias en mayor proporción que la media del sistema cuando la demanda final de todos los países aumenta en una unidad, y que, por lo tanto, se trata de un país con un fuerte efecto de arrastre hacia delante.
Los índices calculados hasta ahora son promedios y, por tanto sensibles a los valores extremos. Los efectos pueden estar concentrados en muy pocos países que dependen en gran medida de otros. Parece, sin embargo, razonable seleccionar como país clave aquellos cuyos efectos se reflejan en muchos países, porque sus efectos se difundirán en mayor medida en el sistema.

Para medir la mayor a menor concentración de los efectos de arrastre se calculan los siguientes índices de dispersión, que recogen los coeficientes de variación (desviación estándar con respecto a la media):

$$
\begin{aligned}
& v_{j}=\frac{\sqrt{\frac{1}{n-1} \sum_{i}\left(z_{i j}-\frac{1}{n} \sum_{i} z_{i j}\right)^{2}}}{\frac{1}{n} \sum_{i} z_{i j}} \\
& v_{i}=\frac{\sqrt{\frac{1}{n-1} \sum_{j}\left(z_{i j}-\frac{1}{n} \sum_{j} z_{i j}\right)^{2}}}{\frac{1}{n} \sum_{j} z_{i j}}
\end{aligned}
$$

A partir de los coeficientes de Rasmussen y sus respectivos coeficientes de dispersión se puede realizar la siguiente clasificación:

- Claves ( $u_{j}>1$ y $u_{i}>1$; vi y $v_{j}$ son bajas). Cuando el efecto de arrastre es superior a la media tanto de un país cualquiera sobre otros países, como de otros países sobre él.

- Estratégico $\left(u_{j}<1\right.$ y $u_{i}>1 ; v_{i}$ bajo). Cuando el efecto de arrastre es inferior a la media de un país cualquiera sobre otros países, pero mayor a ésta, en el efecto de otros países sobre él. La denominación de "estratégicos" apunta al hecho de que son países que pueden constituir posibles estrangulamientos del sistema económico.

- Impulsor de la economía $\left(u_{j}>1\right.$ y $u_{i}<1 ; v_{j}$ bajo). Cuando el efecto de arrastre es superior a la media de un país cualquiera sobre otros países, pero inferior a ésta, en el efecto de otros países sobre él. Es decir, produce efectos mayores sobre la economía que los efectos que se centran en él. 
- Independiente $\left(u_{i}<1\right.$ y $\left.u_{i}<1\right)$. Países para las cuales ambos índices son menores a la media. Son países poco importantes, dado que no provocan efectos de arrastre significativos en el sistema económico, ni reaccionan en forma importante ante el efecto de arrastre provocado por variaciones en la demanda de otros países.

\section{Resultados}

De acuerdo con el cuadro 11, entre los coeficientes de Streit de 2007 y 2013 sobresalen los valores obtenidos por las ligazones entre España-Portugal, lo que indica que existe un verdadero mercado peninsular del tomate.

En el resto de las relaciones, son relevantes las que mantienen los Países Bajos con Alemania, España, el Reino Unido y el resto de los países de la U.E. aunque hayan reducido sus valores en los años de estudio. También destaca la relación Bélgica con Francia.

La relación entre Grecia con el resto de los Países de la U.E. ha pasado a tener una importancia significativa en 2013, ocupando el segundo lugar de los coeficientes de Street.

De acuerdo con el cuadro 12 individualizando la jerarquización por Estados miembros, y utilizando los coeficientes de Chenery-Watanabe, se observa como los Países Bajos se erigen como el Estado miembro con mayores ligazones de importaciones y de exportaciones. Tienen un papel fundamental en el comercio intracomunitario como re-expedidor de frutas y hortalizas al resto de la U.E. (Mellens et al., 2007). El comercio de frutas y hortalizas de este país se centraliza en la empresa The Geenery B.V. Esta inició su andadura en el año 1996 cuando nueve Veiling (subastas con forma jurídica de cooperativas) decidieron unir sus fuerzas con la Oficina Central de Subastas Hortícolas (CBT). La fusión fue impulsada principalmente por el rápido aumento de la escala en el lado de la demanda. Por otra parte el modelo de subasta fue reemplazado por una organización de marketing y ventas con los departamentos auxiliares, entre otras aéreas, de logística, calidad y medio ambiente.

A fin de obtener una tipología de los países en función de los coeficientes de CheneryWatanabe, reflejados en el cuadro 13 , se obtienen los valores de q y $\mathrm{p}$ en relación al promedio del sistema. En este caso los valores son 0,3333 para 2007 y 0,3858 para 2013.

Los Países Bajos, Bélgica, Polonia, Portugal y Francia son claves, en cuanto a sus efectos directos, en el comercio intracomunitario. Tienen un fuerte arrastre tanto en sus exportaciones como importaciones y son fundamentales para enfocar en ellas las presiones de desarrollo, por su mayor capacidad de estimular a otros países.

Los Países Bajos y Bélgica son tradicionales reexportadores en la U.E., no así Portugal y Polonia. Éste último si actúa como re-exportador pero fuera de la UE, y en concreto a la Federación de Rusia.

España y Alemania en 2007 eran claves pero en el año 2013 pasan a ser considerados como Estado con fuerte arrastre el primero (q elevado y $p$ bajo) y Estado base ( $q$ bajo y $p$ alto) el segundo.

El resto de la UE y el Reino Unido son Estados base por sus arrastres hacia atrás (importaciones) en los dos años estudiados. Italia y Grecia, en esos años son Estados independientes ( $q$ y $p$ bajos).

Los Países Bajos presentan los valores mayores de los coeficientes internos en los años de estudio, con Bélgica en el 2013 ocupando el segundo lugar, como se refleja en el cuadro 14.

Al analizar los coeficientes de difusión se aprecia como Países Bajos, Alemania, Bélgica, Reino Unido, y Polonia tienen los valores más significativos. La mayoría ha incrementado 
Cuadro 11. Jerarquización en función del coeficiente de Streit Table 11. Streit coefficients hierarchical

\begin{tabular}{|c|c|c|c|}
\hline Relaciones & 2007 & Relaciones & 2013 \\
\hline España-Portugal & 0,7123 & España-Portugal & 0,6401 \\
\hline Países Bajos-España & 0,3085 & Grecia-Resto U.E. & 0,2831 \\
\hline Países Bajos-Alemania & 0,3076 & Países Bajos-Alemania & 0,2597 \\
\hline Italia-Resto U.E. & 0,2653 & Francia-Bélgica & 0,2596 \\
\hline Francia-Bélgica & 0,2497 & Países Bajos-España & 0,2473 \\
\hline Alemania-Resto U.E. & 0,2263 & Países Bajos-Resto U.E. & 0,2270 \\
\hline Países Bajos-Reino Unido & 0,2093 & España-Francia & 0,2138 \\
\hline España-Francia & 0,1968 & Alemania-Resto U.E. & 0,2109 \\
\hline Reino Unido-Resto U.E. & 0,1889 & Países Bajos-Reino Unido & 0,2039 \\
\hline Países Bajos-Resto U.E. & 0,1821 & Reino Unido-Resto U.E. & 0,1975 \\
\hline Polonia-Resto U.E. & 0,1711 & Países Bajos-Bélgica & 0,1883 \\
\hline Italia-Alemania & 0,1711 & España-Polonia & 0,1735 \\
\hline España-Reino Unido & 0,1612 & España-Reino Unido & 0,1654 \\
\hline España-Polonia & 0,1606 & Polonia-Resto U.E. & 0,1391 \\
\hline Países Bajos-Bélgica & 0,1530 & Italia-Alemania & 0,1343 \\
\hline Francia-Alemania & 0,1288 & Italia-Resto U.E. & 0,1294 \\
\hline Bélgica-Alemania & 0,1195 & Francia-Alemania & 0,1236 \\
\hline Grecia-Resto U.E. & 0,1193 & Bélgica-Alemania & 0,1164 \\
\hline Países Bajos-Italia & 0,1149 & Países Bajos-Italia & 0,1162 \\
\hline Bélgica-Grecia & 0,1106 & España-Alemania & 0,1115 \\
\hline Francia-Italia & 0,1096 & Alemania-Polonia & 0,1020 \\
\hline Polonia-Reino Unido & 0,1076 & España-Resto U.E. & 0,0999 \\
\hline España-Alemania & 0,1054 & Alemania-Grecia & 0,0960 \\
\hline España-Resto U.E. & 0,1018 & España-Italia & 0,0927 \\
\hline Alemania-Grecia & 0,0995 & Países Bajos-Francia & 0,0924 \\
\hline Grecia-Reino Unido & 0,0985 & Francia-Italia & 0,0886 \\
\hline España-Bélgica & 0,0909 & Países Bajos-Polonia & 0,0770 \\
\hline España-Italia & 0,0845 & Italia-Grecia & 0,0685 \\
\hline Alemania-Reino Unido & 0,0675 & Alemania-Reino Unido & 0,0617 \\
\hline Países Bajos-Francia & 0,0617 & Francia-Polonia & 0,0611 \\
\hline
\end{tabular}


Cuadro 11. Jerarquización en función del coeficiente de Streit (continuación) Table 11. Streit coefficients hierarchical (continuation)

\begin{tabular}{|c|c|c|c|}
\hline Relaciones & 2007 & Relaciones & 2013 \\
\hline Bélgica-Resto U.E. & 0,0608 & España-Bélgica & 0,0589 \\
\hline Polonia-Grecia & 0,0599 & Polonia-Reino Unido & 0,0547 \\
\hline Países Bajos-Polonia & 0,0569 & Francia-Resto U.E. & 0,0543 \\
\hline Países Bajos-Grecia & 0,0564 & Bélgica-Resto U.E. & 0,0479 \\
\hline Alemania-Polonia & 0,0542 & Francia-Reino Unido & 0,0397 \\
\hline Francia-Resto U.E. & 0,0498 & Bélgica-Grecia & 0,0393 \\
\hline Francia-Reino Unido & 0,0453 & Polonia-Grecia & 0,0329 \\
\hline Italia-Reino Unido & 0,0404 & Italia-Reino Unido & 0,0298 \\
\hline Francia-Polonia & 0,0403 & Italia-Polonia & 0,0291 \\
\hline Italia-Grecia & 0,0320 & Bélgica-Italia & 0,0267 \\
\hline Alemania-Portugal & 0,0297 & Portugal-Polonia & 0,0203 \\
\hline Italia-Polonia & 0,0173 & Bélgica-Reino Unido & 0,0170 \\
\hline Bélgica-Italia & 0,0142 & Francia-Portugal & 0,0144 \\
\hline Bélgica-Reino Unido & 0,0137 & Portugal-Reino Unido & 0,0124 \\
\hline Bélgica-Polonia & 0,0119 & Alemania-Portugal & 0,0116 \\
\hline Francia-Grecia & 0,0118 & Países Bajos-Grecia & 0,0086 \\
\hline Francia-Portugal & 0,0075 & Bélgica-Polonia & 0,0057 \\
\hline Portugal-Reino Unido & 0,0024 & Italia-Portugal & 0,0025 \\
\hline Países Bajos-Portugal & 0,0012 & Países Bajos-Portugal & 0,0019 \\
\hline Italia-Portugal & 0,0008 & España-Grecia & 0,0014 \\
\hline Bélgica-Portugal & 0,0003 & Bélgica-Portugal & 0,0008 \\
\hline España-Grecia & 0,0001 & Portugal-Resto U.E. & 0,0005 \\
\hline Portugal-Resto U.E. & 0,0001 & Francia-Grecia & 0,0000 \\
\hline Portugal-Polonia & 0,0000 & Portugal-Grecia & 0,0000 \\
\hline Portugal-Grecia & 0,0000 & Grecia-Reino Unido & 0,0000 \\
\hline
\end{tabular}

sus valores en los años de estudio. En el caso de los Países Bajos y Alemania, un aumento de una unidad en su demanda intermedia motivado por un aumento en el consumo aparente traerá como consecuencia un aumento de su demanda final de un 12,70 y 8,57 veces por unidad respectivamente al que lo originó. Y el Estado miembro más beneficiado sería en ambos casos España con un aumento de un $702,14 \%$ y un $429,24 \%$ respectivamente. 


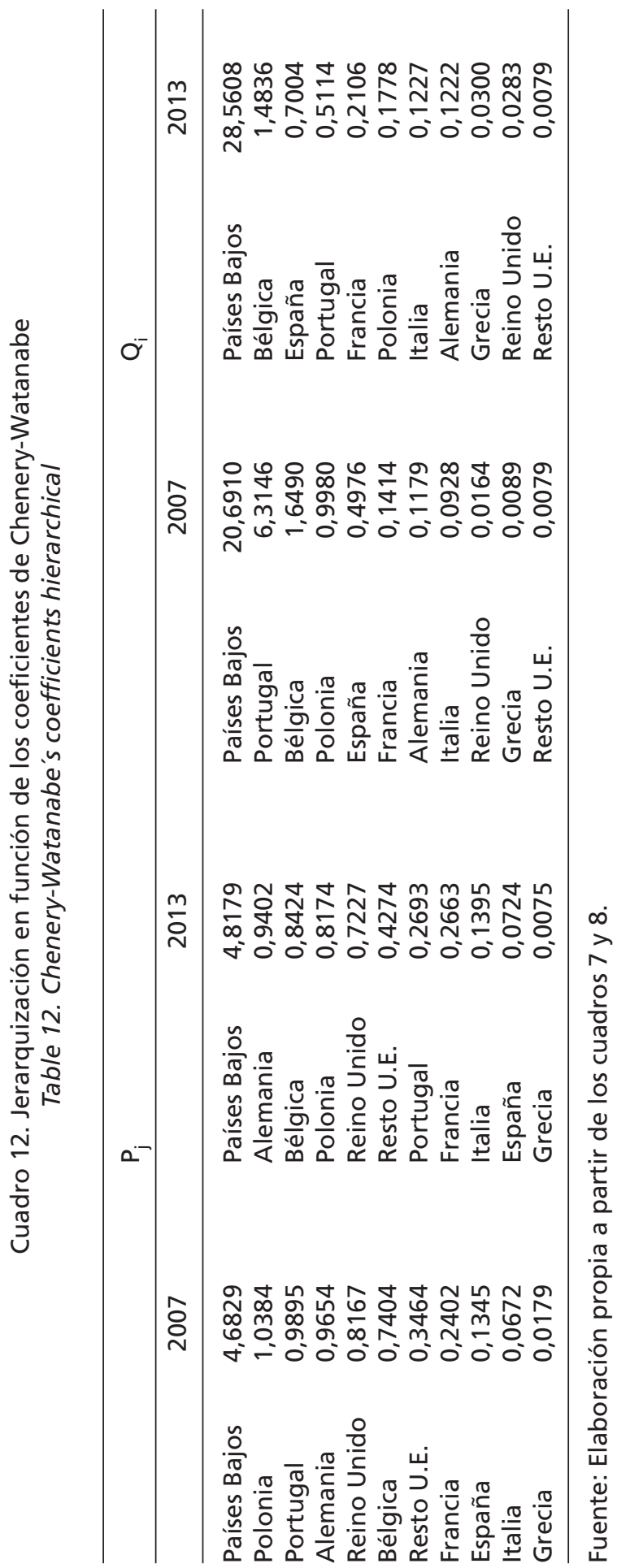

Observando los coeficiente de Rasmussen que vienen en el cuadro 15, se aprecia como en los valores de los coeficientes de absorción $\left(u_{i}\right)$ sobresale España, seguido de Francia, Países Bajos y Bélgica. Portugal en los años de estudio ha sufrido un descenso significativo.

Tanto en 2007 como en 2013, el valor mayor del coeficiente de absorción lo tienen los $\mathrm{Pa}$ íses Bajos y España respecto al coeficiente de poder de difusión.

A partir de los valores de los coeficientes de Rasmussen y sus correspondientes índices de dispersión se puede realizar una jerarquización de los Estados en función de: claves, estratégicos, impulsores de la economía e independientes como se puede observar en el cuadro 16.

De acuerdo con los resultados se consideran claves a los Países Bajos tanto en el año 2007 como en el 2013, no así Bélgica que sólo tiene este calificativo en 2013.

España se caracteriza como estado estratégico en los años de estudio y Francia solamente en 2013.

Alemania, Polonia, Reino Unido y Bélgica se conceptúan como impulsores del sistema en 2007, y en 2013 permanecen los mismos salvó Bélgica.

Francia, Italia, Portugal, Grecia y el resto de países de la U.E. son Estados independientes en 2007. En 2013 permanecen los mismos con la excepción de Francia.

\section{Discusión}

El concepto de "país clave", definido según los coeficientes de Rasmussen, nos permite definir los actores más dinámicos e importantes desde la perspectiva del desarrollo económico. Llama la atención que, en contra de lo que intuitivamente podría parecer 
Cuadro 13. Clasificación en función de los coeficientes de Chenery Watanabe Table 13. Chenery-Watanabe's coefficients classification

\begin{tabular}{lllc}
\hline $\begin{array}{l}\text { Py q elevados } \\
\text { Estados claves }\end{array}$ & \multicolumn{2}{l}{$\begin{array}{l}\text { Q elevado y p bajo } \\
\text { Estados con fuerte arrastre }\end{array}$} \\
\hline 2007 & 2013 & 2007 & 2013 \\
\hline $\begin{array}{l}\text { Países Bajos } \\
\text { Bélgica } \\
\text { Polonia }\end{array}$ & $\begin{array}{l}\text { Países Bajos } \\
\text { Bélgica } \\
\text { Portugal }\end{array}$ & Polonia & España \\
$\begin{array}{l}\text { Francia } \\
\text { Alemania }\end{array}$ & Portugal & & \\
España & Francia & & \\
\hline Q bajo y p elevado & & & \\
Estado Base & & Q bajo y p bajo & \\
\hline 2007 & & Estados independientes \\
\hline Reino Unido & Reino Unido & Italia & Italia \\
Resto U.E. & Resto U.E. & Grecia & Grecia \\
& Alemania & & \\
\hline
\end{tabular}

Cuadro 14. Coeficientes internos de absorción y difusión

Table 14. Absorption and diffusion internal coefficients

\begin{tabular}{lccrrrr}
\hline \multirow{2}{*}{ País } & \multicolumn{2}{c}{ Interno } & \multicolumn{2}{c}{ Difusión } & \multicolumn{2}{c}{ Absorción } \\
\cline { 2 - 7 } & 2007 & 2013 & 2007 & 2013 & \multicolumn{1}{c}{2007} & \multicolumn{1}{c}{2013} \\
\hline Países Bajos & 1,72267 & 1,93870 & 12,7055 & 13,1333 & 4,8370 & 5,8697 \\
España & 1,22545 & 1,12189 & 1,4617 & 1,2794 & 24,7260 & 24,0571 \\
Francia & 1,04419 & 1,11663 & 1,9252 & 2,1385 & 3,2514 & 6,2104 \\
Bélgica & 1,28364 & 1,60500 & 4,9866 & 6,9471 & 4,1329 & 5,5709 \\
Italia & 1,00747 & 1,01073 & 1,4348 & 1,9601 & 1,8519 & 1,6269 \\
Alemania & 1,39118 & 1,20790 & 8,5710 & 8,9033 & 3,2991 & 2,3570 \\
Portugal & 1,18719 & 1,01627 & 3,1103 & 1,4298 & 4,1089 & 2,2268 \\
Polonia & 1,01981 & 1,01926 & 5,5927 & 5,5167 & 1,2925 & 1,2219 \\
Grecia & 1,00003 & 1,00002 & 1,1260 & 1,0461 & 1,0166 & 1,0215 \\
Reino Unido & 1,02811 & 1,05286 & 6,6040 & 6,3752 & 1,2093 & 1,4369 \\
Resto U.E. & 1,01200 & 1,03731 & 3,4377 & 4,3659 & 1,2299 & 1,4965 \\
\hline
\end{tabular}


Cuadro 15. Coeficientes de Rasmussem e índices de dispersión Table 15. Rasmussem's coefficients. and dispersion indexes

\begin{tabular}{lcccccccc}
\hline & \multicolumn{3}{c}{ Coeficientes de Ramussem } & \multicolumn{3}{c}{ Índices de Dispersión } \\
\hline & \multicolumn{3}{c}{$\begin{array}{c}\text { Poder de } \\
\text { dispersión }\left(u_{j}\right)\end{array}$} & \multicolumn{2}{c}{$\begin{array}{c}\text { Sensibilidad de } \\
\text { dispersión }\left(u_{j}\right)\end{array}$} & \multicolumn{2}{c}{ Difusión $\left(v_{j}\right)$} & \multicolumn{2}{c}{ Absorción $\left(v_{j}\right)$} \\
\hline Países Bajos & 2007 & 2013 & 2007 & 2013 & 2007 & 2013 & 2007 & 2013 \\
España & 0,31528 & 2,7209 & 1,0442 & 1,2160 & 0,5511 & 1,4294 & 0,0140 & 0,0683 \\
Francia & 0,2651 & 5,3377 & 4,9840 & 0,2164 & 0,2607 & 1,3717 & 1,2599 \\
Bélgica & 1,0765 & 1,4393 & 0,8922 & 1,1541 & 0,0242 & 0,0931 & 0,0341 & 0,0487 \\
Italia & 0,3097 & 0,4061 & 0,3998 & 0,3370 & 0,2183 & 0,1703 & 0,1898 & 0,2096 \\
Alemania & 1,8503 & 1,8445 & 0,7122 & 0,4883 & 0,2689 & 0,3443 & 0,0910 & 0,1618 \\
Portugal & 0,6714 & 0,2962 & 0,8870 & 0,4613 & 0,1039 & 0,2391 & 0,0357 & 0,1703 \\
Polonia & 1,2073 & 1,1429 & 0,2790 & 0,2532 & 0,0656 & 0,0099 & 0,2280 & 0,2362 \\
Grecia & 0,2431 & 0,2167 & 0,2195 & 0,2116 & 0,2394 & 0,2961 & 0,2468 & 0,2493 \\
Reino Unido & 1,4256 & 1,3208 & 0,2611 & 0,2977 & 0,1346 & 0,0497 & 0,2337 & 0,2221 \\
Resto U.E. & 0,7421 & 0,9045 & 0,2655 & 0,3100 & 0,0815 & 0,0044 & 0,2323 & 0,2182 \\
\hline
\end{tabular}

Cuadro 16. Clasificación en función de los coeficientes de Rasmussen e índices de dispersión Table 16. Rasmussem's coefficients.and dispersion indexes classification

\begin{tabular}{llllll}
\hline \multicolumn{2}{c}{2007} & & & 2013 \\
\hline$u_{i}>1$ & $u_{j}>1$ & $u_{j}<1$ & & $u_{j}>1$ & $u_{j}<1$ \\
\hline$u_{j}<1$ & Países Bajos & España & $u_{i}>1$ & Países Bajos & España \\
& & & Bélgica & Francia \\
& Bélgica & Francia & $u_{j}<1$ & Alemania & Italia \\
& Alemania & Italia & & Polonia & Portugal \\
& Polonia & Portugal & & Reino Unido & Grecia \\
& Reino Unido & Grecia & & & Resto U.E. \\
& & Resto U.E. & & & \\
\hline
\end{tabular}

como más lógico, los dos Estados miembros que se han identificado como "claves" no son los principales productores de tomate, incluso segregando las estadísticas del tomate para industria de las del tomate en fresco.
Tanto los Países Bajos como Bélgica centran su estrategia comercial en la re-exportación de tomate a otros Estados miembros y países terceros, lo cual no es contradictorio con el aumento de la producción nacional. Ambos pro- 
cesos se han se ha intensificado en los últimos años, sobre todo en el caso del primero que es en la actualidad el principal exportador.

Ambos estados han centrado su estrategia en el mercado y en una mejora en su organización comercial. Ejemplo de esa estrategia y mejora fue la eliminación del sistema de subastas a la baja (auctions) en origen (veiling) que estaban siendo poco eficientes en los Países Bajos (Aramyan et al., 2006). Se han reconvertido en la actual "The Greenery"1, la mayor cooperativa y organización de productores (OP) de frutas y hortalizas de la U.E.

Las empresas agrarias de los Países Bajos han centrado su estrategia en productos de calidad (Cantliffe y Vansickle, 2012), en la innovación (Belussi y Sedita, 2008), la tecnología (Diederen et al., 2003) y en el abastecimiento durante todo el año de sus clientes, lo que es esencial hoy en día para la gran distribución. Ese abastecimiento continuo se logra por medio de compras a otros Estados miembros y terceros países. En este sentido, la disposición prevista en la actual reglamentación comunitaria para no considerar como volúmenes comprados a terceros, los adquiridos por una OP europea a otra OP europea a efectos del mantenimiento de su reconocimiento, está diseñada para facilitar esta estrategia.

La trayectoria de España, segundo exportador de la U.E., no ha ido en el mismo sentido. El principal productor europeo de tomate para consumo en fresco, el suministrador clave en los meses de invierno, no es Estado "clave" en el comercio intracomunitario. Los datos nos cuentan que sólo tiene fuertes arrastres hacia adelante y no resulta Estado clave en los años de estudio. Se considera como un Estado estratégico y apunta al he- cho de que pueden constituir posibles estrangulamientos del sistema económico, sobre todo teniendo en cuenta que las zonas productoras son más proclives a crisis de mercado (Gaetano y Cioffi, 2012).

A mediados de la década pasada, España era el líder indiscutible en el mercado intracomunitario (De Pablo Valenciano y Pérez Mesas, 2004). La dotación de factores (clima, mano de obra, etc.) ya no es tan diferencial.

Por un lado, los terceros países de la Cuenca del Mediterráneo ( $y$, en primer lugar Marruecos) tienen algunas ventajas comparativas, sobre todo en el coste de la mano de obra. Pero esto no explica el por qué las dinámicas de producción son divergentes entre España (a la baja) y los Países Bajos (a la alza).

España no ha sido capaz de aprovechar las posibilidades de re-exportar de productos procedentes de Marruecos, como había hecho Francia (mercado de Perpiñán) y Países Bajos con España. Ha habido que esperar al año 2015 para ver a la primera empresa española, CASI, aliarse con la holandesa Norfresh para cerrar un ciclo de 12 meses $^{2}$ y así poder alcanzar la figura de "responsable de compra" de cara a las grandes cadenas de distribución.

A esto se le añade una mala organización comercial que se inicia con el perpetuo problema de la atomización y el mantenimiento con gran importancia de modelos de comercialización pasiva (alhóndigas) frente a modelos comerciales más dinámicos.

Alemania, Polonia, Reino Unido se comportan como Estados impulsores (o motores) con una fuerte eslabonamiento hacia atrás y débil hacia adelante. Hay que tener en cuenta que estamos realizando el análisis intracomunitario, razón por la cual Polonia tiene unos débiles

1. http://en.thegreenery.com/home. Date 10/09/2015.

2. https://joseantonioarcos.wordpress.com/2015/04/03/casi-se-alia-con-la-holandesa-norfresh-para-cerrar-un-ciclode-12-meses/. Date 10/09/2015. 
encadenamientos hacia adelante. Si se hubiera considerado el comercio extracomunitario, se apreciaría la importancia que tienen sus exportaciones a Rusia.

Portugal, Grecia y resto de la U.E no tienen relaciones intensas con otros Estados miembros $y$, por tanto, se consideran independientes.

\section{Conclusiones}

Como se ha visto al principio de este artículo, las expediciones comunitarias, tanto en su vertiente de exportaciones como de importaciones, tiene mucha más importancia que las exportaciones e importaciones de países terceros. Los encadenamientos entre los Estados miembros son, por lo tanto, muy intensos. El análisis input-output nos permite estudiar las ligazones entre los miembros de un sistema (la Unión Europea) para un producto determinado (tomate).

En primer lugar por medio de la matriz de coeficientes se ha analizado los efectos directos que producen esas ligazones en el sistema. Se observa, primero, como por medio de los coeficientes de Streit, España y Portugal actúan como un verdadero mercado peninsular del tomate. Al efectuar la jerarquización por medio de los coeficientes de Chenery- Watanabe, se concluye que los Países Bajos, Bélgica, Polonia, Portugal y Francia pueden ser considerados como claves. Al incorporar los efectos indirectos por medio de la matriz inversa, y reformulando los efectos difusión y absorción, se obtienen los coeficientes de Rasmussen. Se aprecia claramente el papel de los Países Bajos y Bélgica como Países Claves en el comercio intracomunitario del tomate de la Unión Europea.

La desaparición de España en el 2013, comparado con el 2007, de la lista de países claves, demuestra una pérdida de protagonismo comercial preocupante. Después de una época de crecimiento cuantitativo importante, a raíz de la instauración del mercado único y el fin anticipado del periodo de transición prevista en el Acta de adhesión de España a las Comunidades Europeas (el 1 de enero de 1993), el sector tomatero español sufre una crisis de producción primero, pero sobre todo de adaptación a los nuevos tiempos que puede llegar a ser una crisis de identidad.

Como demuestran los casos holandeses y belgas, ventajas comparativas de organización, comercial, de investigación, de innovación pueden compensar con creces otras ventajas comparativas como pueden ser los costes laborales o el acceso a productos fitosanitarios de uso prohibidos en la Unión Europea. El suministrador básico de producto es el que está en peor posición debido a que se está a la espera de que los compradores tengan la bondad de aparecer por las fincas para hacerse cargo del producto. Todo ello en competencia clara con otros suministradores, beneficiándose la gran distribución que cada vez más concentrada.

\section{Bibliografía}

Andreososso-O'Callaghan B, Yue G (2004). Intersectoral linkages and key sectors in China 19871997. Asian Economic Journal 18 (2): 165-183.

Aramyan LH, Ondersteijn CJ, Lansink, AG, van Kooten O, Wijnands JH (2006). Analyzing greenhouse firm performance across different marketing channels. Agribusiness 22(2): 267-280

Belussi F, Sedita SR (2008). The symbiotic division of labour between heterogeneous districts in the dutch and italian horticultural industry. Urban Studies 45(13): 2715-2734.

Bojacá CR, Casilimas HA, Gil R, Schrevens E (2012). Extending the input-output energy balance methodology in agriculture through cluster analysis. Energy 47: 465-470. 
Cantliffe DJ, Vansickle JJ (2012). Competitiveness of the Spanish and Dutch greenhouse industries with the Florida fresh vegetable industry. University of Florida. http://edis.ifas.ufl.edu/pdffiles/CV/CV28400.pdf. Date 03/05/2013.

Chenery H, Watanabe T (1958). International comparisons of the structure of productions. Econometrica 56: 487-521.

Comisión Europea (2014a). Statistics EU trade flows inside \& outside Europehttp://exporthelp. europa.eu/thdapp/display.htm?page=st\%2fst_S tatistics.html\&docType=main \&languageld=en. Date 03/05/2013.

Comisión Europea (2014b). Document de travail. Groupe de travails prévisions tomates. http://ec. europa.eu/agriculture/fruit-and-vegetables/product-reports/tomatoes/index_en.htm. Date 09/09/2015.

De Pablo Valenciano J, Pérez Mesas JC (2004). The competitiveness of Spanish tomato export in the European Union. Spanish Journal of Agricultural Research 2 (2): 167-180.

Diederen P, Van Tongeren F, Van DV(2003). Returns on investments in energy-saving technologies under energy price uncertainty in Dutch greenhouse horticulture. Environmental and Resource Economics 24(4): 379-394.

Gürkan GY, Keçeli S (2009). The intersectoral linkage effects in Turkish economy: an application of static leontief model. PANOECONOMICUS 3 : 301-326.

Hirschman AO (1958). The strategy of economic development. Yale University Press. New Heaven, EE.UU.
Hoekstra R, Edens B, Zult D, Wilting H (2014). Reducing the variation of environmental footprint estimates based on multiregional inputoutput databases. Sustainability Accounting, Management and Policy Journal 5 (3): 325-345.

Jahangard E, Keshtvarz V (2012). Turkey economies: a network theory approach. Iranian Economic Review 16 (32): 45-58.

Keuning SJ, Ruijter, WA (1988). Guidelines to the construction of social accounting matrix. Review of Incomes and Wealth 34(1): 71-100.

Leontief W (1941). The structure of the American economy, 1919-1939. Oxford University Press, Reino Unido.

Miller RE, Blaid PD (1985): Input-Output Analysis: Foundations and Extensions. Prentice Hall. New Jersey, EE.UU.

Olczyk M (2011): Structural changes in the Polish economy - the analysis of input-output. Munich Personal RePec Archive.

Santeramo FG, Cioffi A (2012). Transmission of market crises in the European vegetables sector. Pagri 2/2012 http://ageconsearch.umn.edu/bitstream/150386/2/Santeramo_Cioffi.pdf. Date 09/09/2015.

Streit ME (1969). Spatial associations and economic linkages between industries. Journal of Regional Science 9: 177-188.

(Aceptado para publicación el 23 de diciembre de 2015) 